\title{
Fluid-Signal Structures in the Cervical Spinal Cord on MRI: Anterior Median Fissure versus Central Canal
}

(D)T.A. Tomsick, (DE. Peak, and (D) L. Wang

\begin{abstract}
BACKGROUND AND PURPOSE: Hyperintense fluid-signal anterior median fissure and hyperintense foci resembling the central canal are seen on cervical spine axial T2 MR imaging. They may also be associated with a channel-like T2-hyperintense craniocaudad line on sagittal images. We hypothesized that the hyperintense foci and the sagittal line may represent the base of the anterior median fissure.
\end{abstract}

MATERIALS AND METHODS: In this exploratory study, 358 cervical MR images were analyzed for recording and comparing the incidence/ numbers of hyperintense foci, anterior median fissure, and sagittal line as hyperintense foci, anterior median fissure, and sagittal line per patient when present alone or together, both with and without the sagittal line.

RESULTS: Hyperintense foci were identified on 238/358 (66.5\%) studies; an anterior median fissure, on 218/358 (60.9\%). The hyperintense foci/anterior median fissure ratio was 3.7/2.3 $(P=.00001)$. Anterior median fissures were seen alone less commonly than hyperintense foci were seen alone $(P=.045)$. We identified increased anterior median fissure/patient in a hyperintense foci + anterior median fissure group compared with an anterior median fissure-only group (4.0 versus 3.2, $P=.05$ ), with similar hyperintense foci/patient in the hyperintense foci+anterior median fissure and hyperintense foci-only groups (5.5 versus 5.8, $P=.35$ ), and proportional distribution of both across the hyperintense foci+anterior median fissure subgroups (hyperintense foci/anterior median fissure ratio, 1.3). The sagittal line in 89 (24.9\%) patients was associated with increased hyperintense foci and anterior median fissure/patient. Greater correlation of anterior median fissure/patient to sagittal line presence was seen in sagittal line subgroup analysis.

CONCLUSIONS: This exploratory analysis found an increased anterior median fissure per patient in conjunction with hyperintense foci presence, a proportional increase of both across the hyperintense foci + anterior median fissure group, and greater correlation of anterior median fissure per patient with the sagittal line. These findings suggest that anterior median fissure and hyperintense foci are structurally related, that hyperintense foci may commonly be the base of the anterior median fissure, and that the sagittal line is a manifestation primarily of an anterior median fissure, occasionally appearing as channels that may simulate the central canal.

ABBREVIATIONS: $\mathrm{AMF}=$ anterior median fissure; $\mathrm{CTM}=\mathrm{CT}$ myelography; $\mathrm{HIF}=$ hyperintense foci; $\mathrm{pt} .=$ patient; $\mathrm{SL}=$ sagittal line

A linear midline fluid-signal cleft of the anterior median fissure (AMF) may be seen in the anterior midline cervical spinal cord on T2-weighted fast spin-echo or gradient recalled-echo MR images. Localized midline, fluid-signal may also be seen as hyper-

Received June 21, 2016; accepted after revision November 8.

From the Department of Radiology, University of Cincinnati Academic Health Center, University Hospital, Cincinnati, Ohio.

E. Peak was supported by the Department of Radiology Medical Student Summer Research Program.

Please address correspondence to Thomas A. Tomsick, MD, University of Cincinnati, UC Health, Department of Radiology, 231 Albert Sabin Way, Cincinnati, OH 45267-0761; e-mail: Thomas.Tomsick@UCHealth.com

Indicates article with supplemental on-line tables.

Indicates article with supplemental on-line photo.

http://dx.doi.org/10.3174/ajnr.A5121 intense foci (HIF) on a variety of T2-weighted MR pulse sequences (Fig 1), typically relatively anterior in position, frequently attributed to the central canal. Anatomic sections have confirmed that the base of the anterior median fissure may be wider than its cleft and separated from the central canal by a fraction of a millimeter, and sometimes only by an ependymal/ fiber membrane. ${ }^{1,2}$ MR imaging of spinal cord specimens has depicted a widened base of the AMF as well. ${ }^{3,4}$ In addition, T2hyperintense craniocaudad lines on sagittal images of variable intensity, width, continuity, length, and sharpness may be seen in the anterior aspect of the cord and attributed to the central canal. ${ }^{5}$ While identified on routine clinical imaging, the features may be even more evident on $8 \mathrm{~T}$ high-field, research imaging. ${ }^{6}$

We hypothesize that HIF and AMF are related, the former a manifestation of the latter in some instances, and that either or both may 


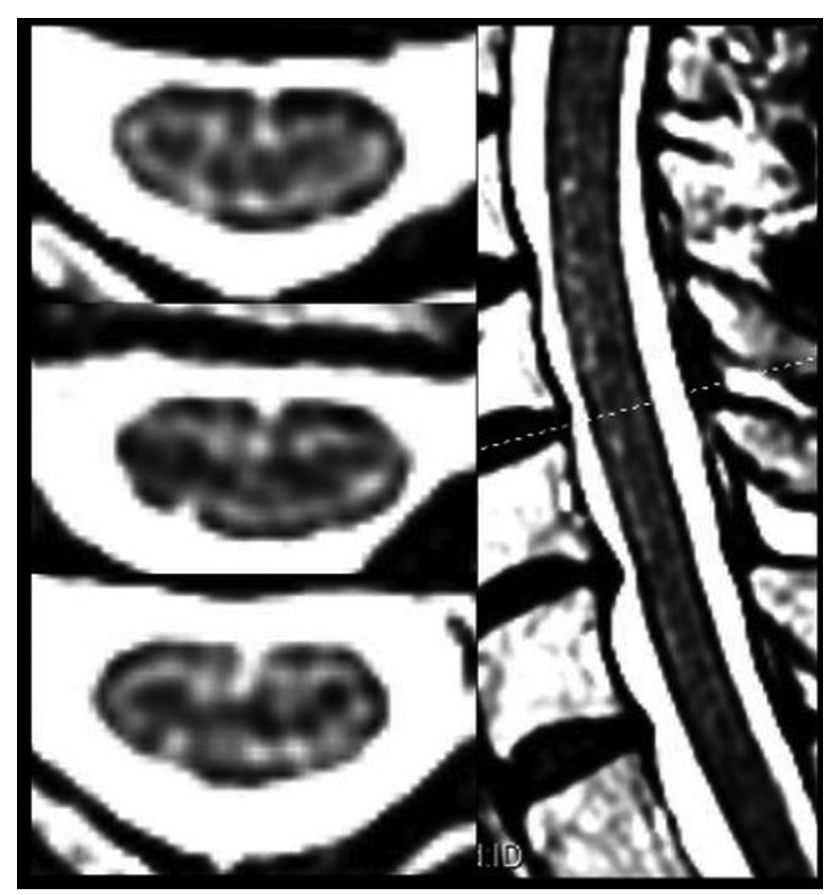

FIG 1. Three consecutive axial FSE-T2 images (left) and a 3-mm midline sagittal image (right). Left, The upper image demonstrates a conspicuous HIF (asymmetrically located to the left) with no AMF. An anterior "dimple" indentation of the cord is typical at the anterior aspect of the AMF but is not considered an AMF unless accompanied by the sagittal cleft as in the lower image. Middle left, An adjacent section demonstrates an HIF continuous with the base of a faint AMF. Lower left, Adjacent section demonstrates an asymmetric, wider, more conspicuous AMF without a definite HIF. Right, FSE-T2WI midline anterior (ventral) sagittal line (SL-MR imaging) is thin and incomplete and of variable length in the anterior $30 \%$ of the cord. Small focal hyperintensities also extend along its course, contributing to variable SL thickness, continuity, and irregularity.

contribute to the craniocaudad lines on sagittal FSE-T2WI (sagittal line $[S L])$. Characterizing the presence and features of the HIF and AMF might contribute to defining their relationships not only to one another but also to lines, channels, or canals identified on midline sagittal images in other disease states, such as Chiari malformation. ${ }^{7}$

\section{MATERIALS AND METHODS}

A retrospective analysis of cervical spine MR imaging of 410 consecutive patients from a clinical scanning population in June and July, 2015, was performed. MR imaging was performed on 6 different instruments from 2 different manufacturers. Patient age, sex, indication, scanner site, and instrument were recorded. Imaging features were analyzed separately by 2 neuroradiologists in conjunction with a second-year medical student who recorded all observations on an Excel spreadsheet (Microsoft, Redmond, Washington) and who performed preliminary data analyses. Imaging features analyzed and recorded are listed in Table 1. HIF and AMF on axial FSE-T2WI were identified as present or absent in each patient, counted sequentially from the mid-C2 level to the C7-T1 disc space in each patient, with approximately $24 \mathrm{sec}-$ tions evaluated over a distance of approximately $90-110 \mathrm{~cm}$. All axial and sagittal images were 3-mm-thick, with variable NEXs and matrices (On-line Table 1). HIF and AMF were
Table 1: Imaging features identified, according to 1.5/3T status

\begin{tabular}{|c|c|c|}
\hline Imaging Feature & Number (\%) & $P$ Value \\
\hline \multicolumn{3}{|l|}{ HIF } \\
\hline Patients & $235(66.2 \%)$ & \\
\hline $1.5 \mathrm{~T}(n=216)$ & $148(68.5 \%)$ & \\
\hline $3 \mathrm{~T}(n=139)$ & $87(62.6 \%)$ & $.33^{\mathrm{b}}$ \\
\hline \multicolumn{3}{|l|}{ AMF } \\
\hline Patients & $214(60.2 \%)$ & \\
\hline $1.5 \mathrm{~T}(n=216)$ & $143(66.2 \%)$ & \\
\hline $3 \mathrm{~T}(n=139)$ & $71(51.1 \%)$ & $.007^{\mathrm{a}}$ \\
\hline Depth ratio of AMF (axial) & 0.31 (range, $0.13-0.48$ ) & $.08^{\mathrm{b}}$ \\
\hline SL & $89(24.9 \%)$ & \\
\hline $1.5 \mathrm{~T}(n=219)$ & $44(20.1 \%)$ & \\
\hline $3 \mathrm{~T}(n=139)$ & $45(32.4 \%)$ & $.009^{\mathrm{a}}$ \\
\hline
\end{tabular}

Note:-NA indicates not applicable.

${ }^{a} \chi^{2}$ test.

${ }^{\mathrm{b}}$ Student $t$ test.

counted for each patient, all patient totals were summed, and the total HIFs and AMFs were divided by the number of patients, with a resulting average across the entire population and multiple subgroups reported and compared as HIF and/or AMF per patient by using the Student $t$ test.

Images were viewed in multiple imaging formats, including up to $\times 3$ magnification, and measurements of structures were performed at the PACS workstation by using the standard annotation distance measurement tool. The position of the HIF in an anteroposterior direction (the depth of the HIF) was measured as the distance from a tangent to the anterior surface of the cord to the HIF divided by the anteroposterior diameter of the cord at the same axial level. The length of the AMF was measured from the same tangent to its deepest portion, divided by cord sagittal diameter. The depth of the SL was measured similarly on sagittal FSE-T2WI from the anterior cord to the sagittal line, divided by the cord anteroposterior diameter at the same level. Total SL length was measured as the sum of $\geq 1$ individual line component.

HIF, AMF, and SL identification versus magnet strength were compared via a $\chi^{2}$ test. Overlap reads of 110 scans by the 2 neuroradiologists for HIF, AMF, and SL identification were compared by using the $\kappa$ statistic.

The study was approved by the local investigational review board at the University of Cincinnati.

\section{RESULTS}

Fifty-two of 410 scans were excluded due to suboptimal spinal cord visualization caused by excessive patient motion or susceptibility artifacts, extensive intramedullary disease, spinal mass, hemorrhage or edema, or Arnold-Chiari malformation. Principal indications were multiple sclerosis $(n=182)$ and cervical spine or disc disease, neck pain, sensory disturbance or radiculopathy, or trauma $(n=176)$. Three hundred fifty-eight patients were included in final analysis; 238 (66.5\%) exhibited HIF, and 218 AMF (60.9\%) (Fig 1).

Two-hundred sixteen $(60.8 \%)$ scans were obtained on 4 different $1.5 \mathrm{~T}$ instruments, and 139 (39.2\%) on 2 different 3T scanners. HIF/AMF scanner data was complete for 355 patients. HIFs were identified in 235/355 (66.2\%) patients; AMF, in 214/355 (60.2\%) patients (Table 1$)$.

AMF and HIF were estimated as $<1 \mathrm{~mm}$ wide in $95.6 \%$ of

AJNR Am J Neuroradiol 38:840-45 Apr 2017 www.ajnr.org 
Table 2: Presence, number, and ratio of HIF/AMF in multiple groups No. (\%) HIF/Pt. AMF/Pt. Ratio HIF/AMF $P$ Value (HIF/AMF) ${ }^{a}$

\begin{tabular}{lrrrrl}
\hline All patients & 358 & 3.67 & 2.33 & 1.57 & .00001 \\
Either HIF, AMF & 100 & & & & \\
$\quad$ HIF only & 60 & 5.8 & - & 1.8 & .001 \\
$\quad$ AMF only & 40 & - & 3.2 & 1.3 & .001 \\
Both HIF + AMF & 178 & 5.5 & 4.0 & 1.3 & .004 \\
$\quad$ AMF+HIF & 87 & 6.5 & 4.8 & 1.3 & .02 \\
No AMF+HIF & 91 & 4.3 & 3.3 & 2.0 & .000001 \\
Sagittal line & 89 & 6.7 & 3.3 & 1.5 & .003 \\
Both HIF+AMF & 65 & 6.5 & 4.3 & 1.50 & $\mathrm{NA}^{\mathrm{c}}$ \\
HIF only & 21 & 8.5 & $\mathrm{NA}$ &. & .01 \\
AMF only & 3 & $\mathrm{NA}$ & 5.6 & 1.5 & .13 \\
Continuous HIF/AMF & 31 & 8.3 & 5.4 & 1.5 &
\end{tabular}

Note:-NA indicates not applicable.

a $T$ test: No./pt. HIF vs No./pt. AMF.

${ }^{b}$ Data incomplete for 3 patients.

'Too few AMF-only to compare.

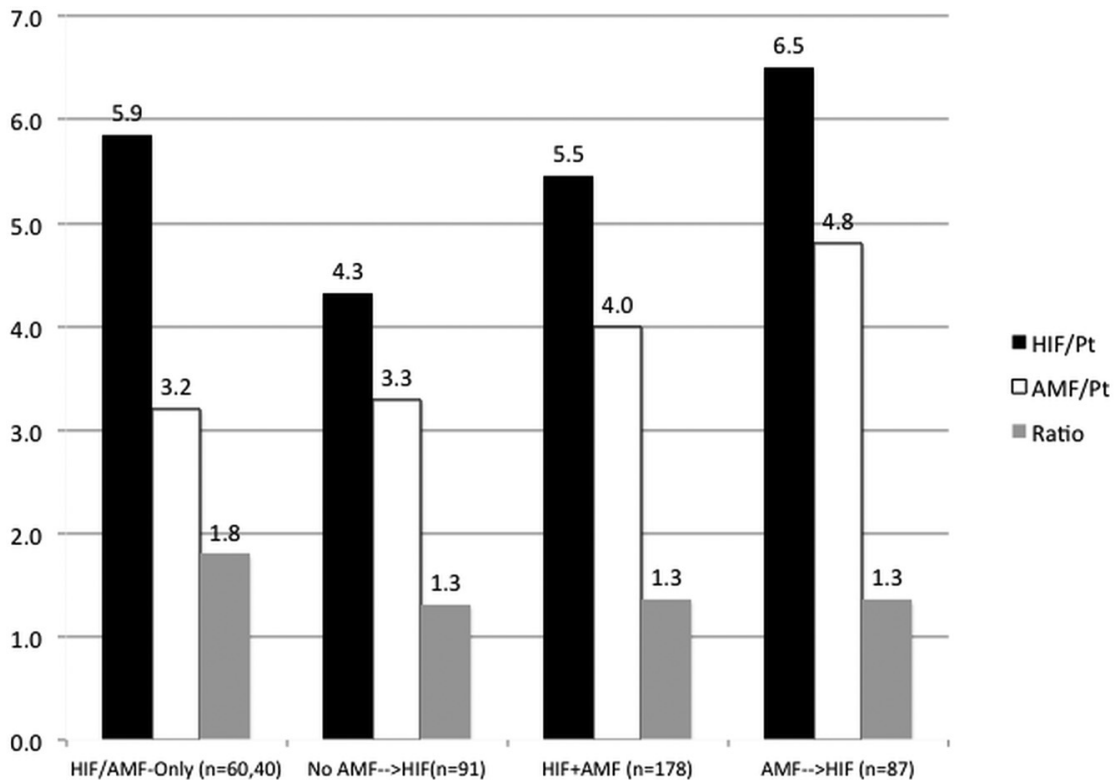

FIG 2. Bar graph comparing HIF/pt., AMF/pt., and HIF/AMF ratios for $100 \mathrm{HIF}$ and AMF-only (left) and the HIF+AMF $(n=178)$ group, flanked by the No AMF $\rightarrow$ HIF subgroup $(n=91)$ to its left, and the AMF $\rightarrow$ HIF $(n=87)$ subgroup to its right.

measured patients. There was no difference between the average HIF and AMF depth ratio ( $0.29 \mathrm{~mm}$ versus $0.28 \mathrm{~mm})$.

The presence, number, and ratio of HIF/AMF in multiple groups with HIF and/or AMF are detailed for comparison in Table 2, including: all patients $(n=358)$; those with either HIF and/or $\operatorname{AMF}(n=278)$; HIF present, with or without $\operatorname{AMF}(n=$ 235); AMF present, with or without HIF ( $n=218)$; both HIF and AMF present (the HIF+AMF group, $n=178$ ); and either HIF $(n=60)$ or AMF $(n=40)$ alone (HIF-only or AMF-only). Data for patients with/without sagittal lines are also included. The baseline HIF/patient (pt.) for all patients $(n=358)$ was greater than the AMF/pt. (3.7 versus 2.3, $P=.000006$ ), with a baseline HIF/AMF ratio of 1.6.

AMFs were less commonly present alone in the absence of HIF, compared with HIF alone $(P=.045)$. When identified alone, the HIF average was 5.8, and AMF, 3.2/pt. (Fig 2). In the $\mathrm{HIF}+\mathrm{AMF}$ group, the AMF average increased to 4.0 greater compared with its presence alone $(P=.05)$, while HIF/pt. remained similar $(5.5)(P=.35)$. In an HIF+AMF subgroup of $91(51.1 \%)$ patients in which no AMF appeared continuous with an HIF, the AMF/pt. was similar to the AMF-only subgroup (3.2 AMFs/pt). In an HIF + AMF subgroup of 87 (48.9\%) patients in which $\geq 1$ AMF appeared inseparable from and continuous with an HIF, the average HIF and $\mathrm{AMF} / \mathrm{pt}$. were 6.5 and 4.8 , respectively. The HIF/AMF mean ratio was 1.3 for the HIF+AMF group and each subgroup.

SLs were present in 89 (24.9\%) patients, always in the presence of HIF and/or AMF (Fig 1 and Tables 1 and 2). Both HIF and AMF were seen more commonly in patients with an SL than without (HIF/pt., 6.7 with SL versus 2.6 without; $P<.0000001$; AMF/pt., 3.3 with SL versus 2.0 without, $P=.002)$. Sixty-five of 89 (73.0\%) SLs were associated with both HIF and AMF (6.5 and $4.4 /$ pt., $P=.003$ ). In 31 patients with SLs and continuous HIF+AMF, the HIF decreased further by comparison with the $\operatorname{AMF}(P=.01)$, with no difference in patients without $\geq 1$ continuous HIF+AMF. SL occurred less frequently with AMF alone $(n=3)$ compared with HIF $(n=21)$, and with only HIF and $\mathrm{AMF} / \mathrm{pt}$. being the greatest in number compared with any group evaluated (8.5 and 5.6, respectively).

Identification of HIF, AMF, and SL varied among scanners. AMFs, but not HIFs, were identified in more patients at $1.5 \mathrm{~T}(P=.006, P=.38$, respectively). SLs were identified more commonly at 3T $(P=.01)$. Agreement between neuroradiologists was substantial for HIF $(\kappa=0.64)$ and $\operatorname{AMF}(\kappa=0.75)$ and moderate for identification of the SL $(\kappa=0.55)$.

\section{DISCUSSION}

In the entire study population, HIFs were identified not only in significantly more scans than AMFs but also in significantly greater numbers per patient when identified. Neither the short distance between the central canal and AMF on spinal cord anatomic cross-section nor even similar average measured depth of the HIF and AMF allows a mere visual impression to represent evidence that the HIF may be a manifestation of an AMF. Despite HIFs being more common than AMFs and greater in number/pt. in the entire scanning population, comparisons of their relative frequencies in multiple settings of isolated and combined presence here support the hypothesis that the HIFs are a manifestation of the AMF in many instances, and not an independent anatomic structure such as the central canal.

$\mathrm{HIF} /$ pt. and AMF/pt. identification when individually present 
(ie, HIF-only and AMF-only) represents baseline averages unaffected by the presence of the counterpart finding, with patients with HIF-only more common than those with AMF-only. Fewer patients with AMF-only are preliminary evidence for a linked, combined presence of HIF and AMF and the MR imaging-identifiable focal dilation of variable diameters at the base of the AMF known to be present anatomically.

In 178 patients in whom both HIF and AMF were present, AMF increased significantly in the presence of HIF from 3.2 to 4.0/pt. ( $P=.05)$, while HIF/pt. remained similar to when it was present alone (5.5 versus 5.8, $P=.35$ ). This increase in AMF/pt. associated with concurrent stable HIF/pt. is more substantial evidence linking HIF and AMF origins.

However, the HIF+AMF group comprised 2 numerically equal subgroups: 1) patients in whom no AMFs have direct continuity with the HIF, and 2) patients with direct visual continuity of the AMF and HIF, in whom $\geq 1$ in an individual patient appears inseparable on imaging. In the former noncontinuous subgroup, mean AMF/pt. is similar to that in the AMF-only groups (3.2/pt.), suggesting a numeric continuum of their relationship to one another as their numbers increase across groups. In the latter $\mathrm{HIF}+\mathrm{AMF}$ subgroup, continuous $\mathrm{HIF}$ and $\mathrm{AMF} / \mathrm{pt}$. increased to 6.5 and 4.8/pt., respectively. Proportional increase across the HIF+AMF group would not be expected as a coincident appearance of 2 unrelated anatomic structures such as the AMF and the central canal but would more likely indicate an anatomic connection of an AMF and an HIF as a manifestation of the same structure. Whereas HIFs were more numerous in the entire population, $\mathrm{HIF} / \mathrm{pt}$. continued to decrease progressively compared with $\mathrm{AMF} / \mathrm{pt}$. when seen alone, when seen in conjunction with one another, and finally when seen as continuous structures. These observations become more than merely consistent with, and further support, a relationship of HIF and AMF, both visually and numerically, reflecting portions of the AMF.

Craniocaudad lines on sagittal imaging have been ascribed to the central canal in $12 / 794(1.5 \%)$ spine MR images, with no consideration for the appearance or potential contribution of the AMF. ${ }^{8}$ Some slit-like "syrinx" cavities of variable width and length have been attributed to a remnant of the central canal seen in a small percentage of adults. ${ }^{5}$ Our analysis of 89 such craniocaudad lines on sagittal imaging here (SL) gives supplementary information regarding potential HIF and SL origins. SLs are seen only in the presence of HIF and/or AMF, eliminating the responsibility of Gibbs artifacts or other normal structures such as the gray matter commissure visible at a high field strength. In the presence of 89 SLs, HIFs are seen in greater numbers/pt. compared with AMFs $(P=.000001)$, but in the 65 patients with SLs with both HIF + AMF, the HIF becomes less significant compared with the $\operatorname{AMF}(P=.003)$. Thiry-one of the 65 patients exhibited $\geq 1$ continuous HIF+AMF, and HIF/pt. decreased further compared with $\operatorname{AMF}(P=.01)$. In 34 patients with SLs with no continuous HIF and AMF, no differences between the 2 were present, but a constant ratio of HIF/AMF was maintained. This progressive, decreased significance of HIF compared with AMF (Table 2), from the entire SL population to the most narrowly defined groups (SL with or without continuous HIF + AMF), with a constant ratio across the group, strengthens the proposal that SLs are more intimately related to the AMF presence. The presence of an $\mathrm{SL}$, or apparent channel, attributable in greater part to AMF rather than HIF presence, reduces any perception that a sagittal channel must be related to the central canal and further suggests an etiologic link between the AMF and HIF.

That SL identification occurred with $21 \mathrm{HIFs}$ and 3 AMFs alone does not negate the importance of an AMF in SL creation, rather indicating that AMFs are less uncommonly seen in the absence of HIF with SL than in HIF without AMF, indicating a close link of the AMF-HIF presence to the SL. SLs are seen with either HIF or AMF alone only when either exhibits a higher average number than seen in any other subgroups analyzed. Few SLs seen with AMF alone indicate the presence of an identifiable focal dilation (HIF) at the AMF base in most instances. Greater numbers of HIF/pt. in the absence of AMFs on MR imaging remain consistent with the presence of AMF known to exist anatomically in each patient, if not identifiable on MR imaging in all.

Additionally, in a separate analysis, we identified and compared HIFs and AMFs on cervical MR imaging versus CT myelography (CTM) in 34 patients; 50 (48.5\%) of 103 AMFs seen on MR imaging were also identified at the same vertebral level on CTM (On-line Table 2), indicating a moderate correlation for AMF identification between the 2 modalities (On-line Fig 1). ${ }^{9}$ Of the 191 HIFs identified on MR imaging, 28 (14.7\%) were associated with an HIF on CTM at the same vertebral level, in the absence of an AMF on MR imaging (On-line Fig 2). Identification of an HIF as a dilation at the base of an AMF on CTM, while failing to identify the AMF on MR imaging, is strong evidence that HIF on MR imaging may represent the base of an AMF. HIFs on CTM were seen alone infrequently, usually associated with AMFs on CTM. Variable demonstration of AMF on MR imaging and/or CTM may be explained by a narrow AMF in some instances or its pia mater lining in others concealing or replacing fluid, while CSF in the subarachnoid space may still circulate and fill the AMF base from communications at higher or lower spinal levels. ${ }^{10}$

A demonstrated SL may be long or short, thin and sharp, or less typically broad and less distinct, paralleling the appearance of an HIF and/or an AMF seen on axial images, typically less than 1 mm wide (Table 1, Fig 1, and On-line Fig 3). The AMF may be uncommonly wide on axial images, more so at their base, simulating an HIF, and associated SLs may be less sharp and detailed, giving an indistinct bandlike appearance related to partial volume effects that are clearly caused by the AMF. Such wider AMFs and HIFs allow some SLs to appear as wide, indistinct channels on sagittal images due to partial volume effects (Fig 3). We wondered whether some channels may then simulate the central canal and thereby simulate hydromyelia when dilated to a subjectively greater degree (On-line Figs 3 and 4). It is hypothesized that a disturbance or alteration of CSF distribution and flow dynamics, in which wider AMFs and associated HIFs become variously confluent and/or segmented in the process, even wider in the AMF base than its sagittal cleft, occurs in some instances, creating the channel and hydromyelia appearance.

A link of the HIF to the AMF and of both to SL on routine clinical MR imaging may then have its greatest significance in SL channels simulating hydromyelia in certain disease processes. For example, thin sagittal channels with greater CSF width, length, 


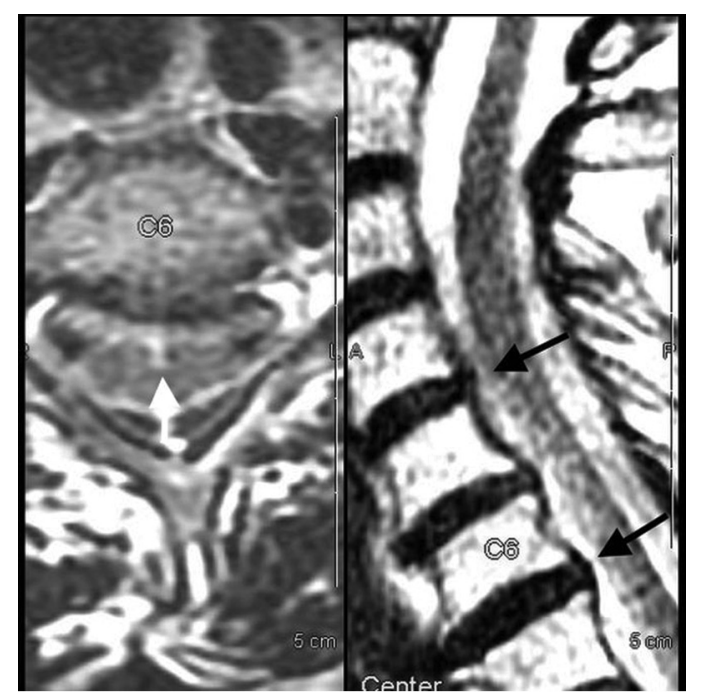

FIG 3. Left, $A M F,>1-m m$-wide (white arrow), extends across the segment from C4-5 to C5-6. The AMF appears to extend into a wider HIF. Right, Indistinct anterior margin of the cord is present at C4 -5 to C5-6 (black arrows) due to partial volume averaging of the wide AMF, sharper above $\mathrm{C} 4$ and below C6.

and volume than identified in our study population, accompanied by prominent HIFs and AMFs on axial images, have been seen in the Arnold-Chiari population, well-known for development of hydromyelia and syringohydromyelia (On-line Figs 3 and 4$)$.

Limitations of our exploratory analysis include the subjective determination of HIF, AMF, and SL presence on a variety of scanning instruments of variable performance, making observations further hypothesis-generating. While interpretations were accepted on the basis of single-reader observations, interobserver agreement was substantial for HIF/AMF and moderate for SL for random double-read cases, perhaps diminished by differences in scanner ability to demonstrate HIF, AMF, and SL. When scanners that performed worst overall were removed from global analysis, analysis of measured HIF and AMF incidence and frequency supported the primary observations.

Difficulty identifying AMF and HIF in the presence of intramedullary disease such as multiple sclerosis likely underestimated the HIF/AMF frequency. HIF/AMF quantitation was a 2D method that did not consider or adjust for the total number or length of axial sections used for analysis, so that quantification of frequency may also be underestimated. Certain cases were excluded due to excessive lesion burden, but most cases were of sufficient quality to quantitate HIF and AMF, even if underestimated.

It might be suggested that presence of both HIF and/or AMF with SL may appear related, but not causative. Identification related to other pathology such as atrophy, as might be seen in older patients or those with MS, may also play a part. ${ }^{11}$ While atrophy might increase the absolute incidence and frequency of either or both HIF and AMF, it would not be expected to impact the comparisons of relative incidence and frequency and the hypotheses proposed here.

The results of this initial explorative analysis warrant a detailed, prospective study capable of confirming the observations and furthering the hypotheses made here. Patients scanned on poorer performing instruments should not be included in future prospective analysis. The number of HIFs and AMFs should be compared not according to the number of patients examined but according to the number of axial sections actually evaluated, so that both nondiagnostic sections with artifacts and variations in patient size may be accounted for. A prospective study might include additional analysis of 2D gradient recalled-echo or FIESTA sequences or dynamic CSF phase-contrast studies to examine CSF pulsation (On-line Fig 5). More detailed measurement of distances and widths of structures of interest on a separate workstation, including one with higher resolution capability, may still not distinguish HIF and the central canal due to their inherent proximity. Subgroup comparisons of various clinical indications may distinguish groups more likely to demonstrate a greater number of HIF, AMF, and SL depictions.

A prospective study of populations known to exhibit prominent canals/channels is warranted, to include patients with Arnold-Chiari malformation. It is hypothesized that HIF and AMF cause a prominent SL mimicking a channel or hydromyelia, which may be related to altered CSF pulsation dynamics known to occur with Arnold-Chiari. Preliminary observation suggests that patients with Arnold-Chiari may have more numerous and conspicuous HIFs and AMFs on axial images and craniocaudad lines or channels on sagittal images.

\section{CONCLUSIONS}

HIF, AMF, and SL are commonly identified as fluid-signal structures on cervical MR imaging, variably observed among scanning instruments of different imaging parameters. Comparisons of HIF and AMF frequency with either or both present, with and without AMF continuity with HIF, indicate that AMFs tend to occur in conjunction with HIFs. While both HIF and AMF are associated with craniocaudad lines on sagittal images, AMFs have a stronger link to these channels. These observations, supported by a parallel comparison of MR imaging and CT myelography, suggest that HIFs are the base of the AMFs in many instances, and not necessarily a separate independent structure (ie, the central canal). We further hypothesize that these channels will occasionally be sufficiently conspicuous to mimic a visible or dilated central canal or hydromyelia.

Disclosures: Elianna Peak—RELATED: Grant: University of Cincinnati Department of Radiology, Comments: summer grant for medical student research.

\section{REFERENCES}

1. Truex RC, Carpenter MB. Internal structure of the spinal cord. In: Truex RC, Carpenter MB, Strong OS. Strong and Elwyn's Human Neuroanatomy. Baltimore: Williams and Wilkins; 1964:187-91, 20708,220

2. Afifi AK, Bergman RA. Spinal cord. In: Afifi AK, Bergman RA. Functional Neuroanatomy: Text and Atlas. New York: McGraw-Hill;1998:63

3. Solsberg MD, Lemaire C, Resch L, et al. High-resolution MR imaging of the cadaveric human spinal cord: normal anatomy. AJNR Am J Neuroradiol 1990;11:3-7 Medline

4. Cavlin MJ, Asato R, Hackney DB, et al. High-resolution MR of the spinal cord in humans and rats. AJNR Am J Neuroradiol 1989;10: 13-17 Medline

5. Holly, LT, Batzdorf U. Slitlike syrinx cavities: a persistent central canal. J Neurosurg 2002;97:161-65 Medline 
6. Zhao W, Cohen-Adad J, Polimeni JR, et al. Nineteen-channel receive array and four-channel transmit array coil for cervical spinal cord imaging at 7T. Magn Reson Med 2014;72:291-300 CrossRef Medline

7. Wang L, Tomsick T: Focal fluid signal change in the spine: anterior median fissure or central canal: anatomical and clinical considerations. In: Proceedings of the Annual Meeting of the American Society of Neuroradiology and the Foundation of the ASNR Symposium, Montreal, Quebec, Canada. May 17-22, 2014

8. Petit-Lacour MC, Lasjaunias $P$, Iffenecker C, et al. Visibility of the central canal on MRI. Neuroradiology 2000;42:756-61 CrossRef Medline

9. Perez V, Peak E, Wang L, et al. MR vs CT myelography in demonstrating normal CSF structures of the cervical spinal cord. In: Pro- ceedings of the Annual Meeting of the American Society of Neuroradiology and the Foundation of the ASNR Symposium, Washington, DC. May 21-26, 2016

10. Jacquesson T, Streichenberger N, Sindou M, et al. What is the dorsal median sulcus of the spinal cord? Interest for surgical approach of intramedullary tumors. Surg Radiol Anat 2014;36:345-51 CrossRef Medline

11. Peak E, Wang L, Tomsick TA. Central canal and/or anterior median fissure: MR imaging in multiple sclerosis patients versus control. In: Proceedings of the Annual Meeting of the American Society of Neuroradiology and the Foundation of the ASNR Symposium, Washington, DC. May 21-26, 2016 